Article

\title{
Distributed Control of Heat Conduction in Thermal Inductive Materials with 2D Geometrical Isomorphism
}

\author{
Chia-Yu Chou ${ }^{1}$, Boe-Shong Hong ${ }^{1, *}$, Pei-Ju Chiang ${ }^{1}$, Wen-Teng Wang ${ }^{2}$, Liang-Kuang Chen ${ }^{3}$ \\ and Chia-Yen Lee ${ }^{4}$
}

1 Department of Mechanical Engineering, National Chung Cheng University, Chia-Yi 62102, Taiwan; E-Mails: yeraeye@gmail.com (C.-Y.C.); pchiang@ccu.edu.tw (P.-J.C)

2 Department of Mechanical and Computer-Aided Engineering, National Formosa University, Yunlin 63201, Taiwan; E-Mail: wtwang@nfu.edu.tw

3 Department of Mechanical Engineering, National Taiwan University of Science and Technology, Taipei 10607, Taiwan; E-Mail: 1kchen@mail.ntust.edu.tw

4 Department of Vehicle Engineering, National Pingtung University of Science and Technology, Pingtung 91201, Taiwan; E-Mail: leecy@mail.npust.edu.tw

* Author to whom correspondence should be addressed; E-Mail: imehbs@ccu.edu.tw; Tel: +886-5-2720411 25 (ext. 33321); Fax: +886-5-2720589.

Received: 21 May 2014; in revised form: 19 August 2014 / Accepted: 29 August 2014 / Published: 15 September 2014

\begin{abstract}
In a previous study we provided analytical and experimental evidence that some materials are able to store entropy-flow, of which the heat-conduction behaves as standing waves in a bounded region small enough in practice. In this paper we continue to develop distributed control of heat conduction in these thermal-inductive materials. The control objective is to achieve subtle temperature distribution in space and simultaneously to suppress its transient overshoots in time. This technology concerns safe and accurate heating/cooling treatments in medical operations, polymer processing, and other prevailing modern day practices. Serving for distributed feedback, spatiotemporal $H_{\infty} / \mu$ control is developed by expansion of the conventional $1 \mathrm{D}-H_{\infty} / \mu$ control to a $2 \mathrm{D}$ version. Therein $2 \mathrm{D}$ geometrical isomorphism is constructed with the Laplace-Galerkin transform, which extends the small-gain theorem into the mode-frequency domain, wherein 2D transfer-function controllers are synthesized with graphical methods. Finally, 2D digital-signal processing is programmed to implement $2 \mathrm{D}$ transfer-function controllers, possibly of spatial fractionorders, into DSP-engine embedded microcontrollers.
\end{abstract}


Keywords: distributed control; $L_{2}$-gain control; $\mathrm{nD}$ transfer functions; thermal inertia

\section{Introduction}

In the works $[1,2]$ and the references cited therein, we learned that many materials possess measurable entropy-flow storage, and that heat conduction in these thermally inductive materials behaves as a standing wave whenever the heat conduction region is small enough to some extent of engineering practice. Known from modal decomposition of standing waves, modes of larger (smaller) variations in space have faster (slower) motions in time. Therefore, tracking of spatially subtle distributions of temperature under feedback control with pointed actuation will always be accompanied by temporally abrupt transients, resulting in unsafe or inaccurate heating/cooling treatments in medical operations, polymer processes, and other prevailing modern day practices.

To remedy such a situation, this paper continues to develop distributed control for non-Fourier heat conduction, inasmuch as distributed sensors and actuators are well-developed nowadays [3-14]. Distributed control is capable of tracking precise local-temperatures with slow heating over the entire region of heat conduction, which is otherwise beyond the nature of pointed control.

The systems \& control literature documents several kinds of feedback technologies applicable to distributed control of non-Fourier heat conduction, which are collected in Table 1. Listed in the first entry are finite-dimensional feedback syntheses based on order-truncated plants, for examples [15-19], which in essence belong to 1D control methodology. For non-Fourier heat conduction, we consider them inadequate to track the robust performance specified both in space and in time. The second and third entries concern infinite dimensionality, wherein Grabowski, Desoer, Callier, Winkin [20-23], and others have adequately developed spectral factorization, algebra of transfer functions and semigroup theories applicable to control purposes. In practice, an infinite-dimensional transfer function from pointed input to pointed output can further be identified in the frequency domain with fraction order [24-28], serving for 1D$H_{\infty} / \mu$ feedback loopshaping. Unfortunately, these dimension-infinitely elegant tools were not originally developed for distributed sensing and actuation.

The fourth entry is suitable for a distributed control strategy, where the $\mathrm{nD}$ state-space robust control [29-34] is extended from 1D robust state-space synthesis to an $\mathrm{nD}$ version in the space-time domain. Controllers from state-space syntheses have spatiotemporal orders close to that of the generalized plant, usually resulting in either order-overabundance or order-shortage in that feasible controllers merely exist in much lower orders or in higher orders, respectively. Since microcontrollers that implement feedback control have limited computation and memory capability, high-order controllers will retard on-line processing of dynamics. Partly for this reason, we develop distributed $H_{\infty} / \mu$ loopshaping to synthesize 2D transfer-function controllers of proper orders, listed in the last entry of Table 1 . In this method, the generalized plant composed of the nominal plant, performance weightings and modelling uncertainty bounds is identified or specified in a mode-frequency domain, served for $2 \mathrm{D}-\mu$ loopshaping in the modefrequency domain for robust performance both in time and in space. It is extended from the conventional $H_{\infty} / \mu$ loopshaping [35] in the frequency domain to $2 \mathrm{D}$ version in mode-frequency domain, named $2 D$ $H_{\infty} / \mu$ loopshaping. 
The so-called 2D transfer-function above is a functional representation of spatiotemporal dynamics through 2D integral transforms, with two independent variables representing the space and time, respectively. The systems \& control literature documents several kinds of 2D integral transform, as listed in Table 2. Therein, the Double Laplace transform, for example in [36,37], and Double Fourier transform, for example in $[15,17,19]$, were always applied for signal processing and feedback control in semi-infinite and infinite space regions, respectively. The Laplace-Galerkin transform [1,38-40] or Fourier-Galerkin transform $[41,42]$ is justified to model the non-Fourier heat conduction and its controllers for bounded space regions that are of real concern in heat conduction practice. Such an integral transform is obtained through the composite of Laplace transform in time and modal decomposition in space. With the Laplace-Galerkin transform, Hong [42] developed the 2D-geometric isomorphism for 2D- $\mu$ loopshaping in mode-frequency domain. Later on, Hong et al. [2] applied 2D transfer-function modelling to prove that the time-delayed heat condition is an ill-posed description that contradicts the first law of thermodynamics. For convenient computation in $2 \mathrm{D}$ or $3 \mathrm{D}$ space regions, the 2D transfer functions can be numerically treated as 3D or 4D transfer-functions, respectively.

Table 1. Candidate types of control for non-Fourier heat conduction.

\begin{tabular}{|c|c|c|}
\hline Methodology & Typical references & Critical comments \\
\hline $\begin{array}{l}\text { 1D control } \\
\text { technology }\end{array}$ & $\begin{array}{l}\text { Bamieh et al. [15] } \\
\text { Stewart et al. [17] } \\
\text { Gorinevsky et al. [19] }\end{array}$ & $\begin{array}{l}\text { - Finite-dimensional controllers based on order-truncated } \\
\text { plants; thus, it is hard to track robust performance imposed on } \\
\text { the originally distributed parameter plants. }\end{array}$ \\
\hline $\begin{array}{l}\text { Infinite transfer } \\
\text { function }\end{array}$ & $\begin{array}{l}\text { Callier and Desoer [20] } \\
\text { Callier and Winkin [21] } \\
\text { Callier et al. [22] } \\
\text { Grabowski and Callier [23] }\end{array}$ & $\begin{array}{l}\text { - Having been theoretically well-developed for classical control } \\
\text { systems with pointed sensing and pointed actuation. }\end{array}$ \\
\hline $\begin{array}{l}\text { Fraction-order } \\
\text { dynamics }\end{array}$ & $\begin{array}{l}\text { Podlubny [24] } \\
\text { Valerio and Costa [25] } \\
\text { Vinagre and Feliu [26] } \\
\text { Monje et al. [27] } \\
\text { Padula and Visioli [28] }\end{array}$ & $\begin{array}{l}\text { - An attractively empirical tool for feedback loopshaping with } \\
\text { pointed sensing and actuation; usually being used in } \\
\text { conjunction with frequency-domain identification. } \\
\text { - Requiring further developments on internal stability issues. }\end{array}$ \\
\hline nD state space & $\begin{array}{l}\text { Valcher [29-30] } \\
\text { Fornasini and Valcher [31] } \\
\text { Shu et al. [34] } \\
\text { Lancaster and Zaballa [32] } \\
\text { Carlos and Anaya [33] }\end{array}$ & $\begin{array}{l}\text { - Extension of } 1 \mathrm{D} \text { robust state-space synthesis upon } \mathrm{nD} \\
\text { discrete plant for } \mathrm{nD} \text { discrete controller. } \\
\text { - Belonging to distributed control technique usually resulting in } \\
\text { either overabundance or shortage of controller orders. }\end{array}$ \\
\hline $\begin{array}{l}\text { Distributed } \\
H_{\infty} / \mu \\
\text { loopshaping }\end{array}$ & Hong [42] & $\begin{array}{l}\text { - Being 2D-version loopshaping upon continuous } \mathrm{nD} \text { plant for } \\
\text { 2D transfer function controllers. } \\
\text { - Graphically finding all feasible controllers with robust } \\
\text { performance. } \\
\text { - Facilitating the identification of generalized plant in mode- } \\
\text { frequency domain. }\end{array}$ \\
\hline
\end{tabular}


Table 2. 2D transfer function modelling.

\begin{tabular}{|c|c|c|}
\hline Methodology & Typical documents & Critical comments \\
\hline $\begin{array}{l}\text { Double Laplace } \\
\text { transform }\end{array}$ & $\begin{array}{l}\text { Dahiya and Vinayagamoorthy [36] } \\
\text { Eltayeb and Kilicman [37] }\end{array}$ & $\begin{array}{l}\text { - Suiting for semi-infinite space regions with initial } \\
\text { conditions in space and time. }\end{array}$ \\
\hline $\begin{array}{l}\text { Double Fourier } \\
\text { transform }\end{array}$ & $\begin{array}{l}\text { Bamieh et al. [15] } \\
\text { Stewart et al. [18] } \\
\text { Gorinevsky et al. [19] }\end{array}$ & $\begin{array}{l}\text { - Being applicable to signal processing and feedback } \\
\text { loopshaping in two-side infinite space regions. }\end{array}$ \\
\hline $\begin{array}{l}\text { Laplace-Galerkin } \\
\text { transform }\end{array}$ & $\begin{array}{l}\text { Rabenstein and Trautmann [38-39] } \\
\text { Hong [42] } \\
\text { Hong et al. [2] }\end{array}$ & $\begin{array}{l}\text { - On bounded space regions has it been well justified to } \\
\text { work. }\end{array}$ \\
\hline $\begin{array}{l}\text { Fourier-Galerkin } \\
\text { transform }\end{array}$ & Hong [42] & $\begin{array}{l}\text { - A 2D geometrical isomorphism for distributed } H_{\infty} / \mu \\
\text { loopshaping on bounded regions }\end{array}$ \\
\hline
\end{tabular}

\section{2D Transfer-Function Modelling}

Consider the non-Fourier heat conduction in a bounded region $\Omega$. It is governed by energy conservation:

$$
\rho C_{v} \frac{\partial T}{\partial t}+\nabla \cdot \boldsymbol{q}=Q
$$

and the non-Fourier law:

$$
R \boldsymbol{q}+L \frac{\partial \boldsymbol{q}}{\partial t}=-\nabla T
$$

where the thermal inductance $L$ is included to fulfill the electro-thermal analogy [1]. Apart from thermal inductance $L$, a differential volume of $\Omega$ is of thermal resistance $R$ and thermal capacitance $C=\rho C_{v}$, where $\rho$ stands for mass density and $C_{v}$ for specific heat at constant volume.

Substituting Equation (2) into Equation (1) yields a hyperbolic equation:

$$
\tau \frac{\partial^{2} T}{\partial t^{2}}+\frac{\partial T}{\partial t}-\frac{1}{C} \nabla \cdot(k \nabla T)=\frac{1}{C}\left(\tau \frac{\partial Q}{\partial t}+Q\right) .
$$

wherein $k$ stands for the thermal conductance, $k \equiv 1 / R$, and $\tau$ for the relaxation time in heat diffusion $\tau \equiv L / R$, with the following assumptions in practice:

(A1) the first-order spatial derivative of the thermal conductance $k$ exists, and

(A2) the nominal value of relaxation time in heat diffusion $\tau$ is uniformly distributed over the operation region $\Omega$.

Let the temperature distribution and heat-rate source be scaled by a reference temperature $\bar{T}$ as $\psi \equiv T / \bar{T}$ and $q \equiv Q / C \bar{T}$, and then we can rephrase the hyperbolic heat-conduction to be:

$$
\tau \frac{\partial^{2} \psi}{\partial t^{2}}+\frac{\partial \psi}{\partial t}-\frac{1}{C} \nabla \cdot(k \nabla T)=\tau \frac{\partial q}{\partial t}+q, \text { in } \Omega \times \Re^{+}
$$

where $q$ represents the dimensionless entropy-rate flowing into the control region. The following boundary conditions are considered: 


$$
\alpha \psi+\beta \nabla \psi \cdot \hat{n}=0 \text { on } \partial \Omega,
$$

with $\alpha(x) \cdot \beta(x) \geq 0$ for all $x \in \partial \Omega$, which includes von-Neumann, Dirichlet, and Robin homogeneous boundary conditions.

The dynamics governed by Equations (3) and (4) involves the non-uniform operator $\boldsymbol{a}$ defined over the set of spatial functions $\phi$ 's $\in L_{2}(\Omega)$ :

$$
\begin{gathered}
\boldsymbol{a}_{\phi}=-(1 / C) \nabla \cdot(k \nabla \phi) \text { in } \Omega, \\
\alpha \phi+\beta \nabla \phi \cdot \hat{n}=0 \text { on } \partial \Omega .
\end{gathered}
$$

Based on the Sturm-Liouville theory [43], the eigenvalues of the non-uniform operator $\boldsymbol{a}$ are all positive, and its eigenfunctions can constitute an orthonormal and complete basis of $L_{2}(\Omega)$. In the sequel, we denote by $\Lambda$ the countable set of $\boldsymbol{a}$ 's eigenvalues and by $\left\{\phi_{\lambda}\right\}_{\lambda \in \Lambda}$ the set of corresponding eigenfunctions.

With respect to the real, countable, feasible, orthonormal and complete eigenfunctions set $\left\{\phi_{\lambda}\right\}_{\lambda \in \Lambda}$, the Laplace-Galerkin transform $\mathscr{H}$ is defined by:

$$
F(\lambda, s) \equiv \mathscr{H}[f(x, t)]=\int_{0^{-}}^{\infty} \int_{\Omega} e^{-s t} C(x) \phi_{\lambda}(x) f(x, t) d x d t .
$$

Here the domain of the one-side Laplace transform, denoted by $\Gamma$, is an infinite line parallel to the imaginary axis, where the integral in Equation (7) converges. Accordingly, the inverse Laplace-Galerkin transform $\mathscr{H}^{-1}$ is:

$$
f(x, t) \equiv \mathscr{H}^{-1}[F(\lambda, s)]=\frac{1}{2 \pi j} \sum_{\lambda \in \Lambda} \int_{\Gamma} F(\lambda, s) \phi_{\lambda}(x) e^{t s} d s .
$$

The Laplace-Galerkin transform $\mathscr{H}$ is of the property:

$$
\mathscr{H}\left[h\left(\boldsymbol{a}, \mathscr{D}_{t}\right) f(x, t)\right]=h(\lambda, s) \cdot \mathscr{H}[f(x, t)],
$$

where $h$ is a ratio of two expressions of finite or some infinite length constructed from two independent variables, one standing for space and the other for time, allowing for the operations of addition, subtraction, multiplication, integer exponents in time, and fraction-order exponents in space. For example:

$$
\mathscr{H}\left[\frac{\mathfrak{D}_{t}-\boldsymbol{a}^{1 / 2}}{\mathfrak{D}_{t}+\boldsymbol{a}^{1 / 2}} f(x, t)\right]=\frac{s-\sqrt{\lambda}}{s+\sqrt{\lambda}} F(\lambda, s) .
$$

Then, performing the Laplace-Galerkin transform $\mathscr{H}$ on Equations (4) and (5) yields a functional representation of the non-Fourier heat conduction, named by the $2 D$ transfer-function:

$$
G(s, \lambda)=\frac{\tau s+1}{\tau s^{2}+s+\lambda} .
$$

As for the feedback controller $K: \Omega \times \Gamma \rightarrow \mathcal{C}$, it can be a more general dynamics:

$$
K(\lambda, s)=\frac{N(\lambda, s)}{M(\lambda, s)}
$$


where $M$ and are of temporal integral-order of $s$ and allow for spatial fraction-order of $\lambda$, but the temporal order of $N$ is not larger than that of $M$. The loop gain $L_{O}$ of the feedback dynamics interconnected by the plant $G$ and the controller $K$ is defined as usual by $L_{O}(\lambda, s) \equiv K(\lambda, s) G(\lambda, s)$.

\section{2D Small Gain Theorem}

In this section, we abstract the contents in [42] as the theoretical background of the 2D- $\mu$ loopshaping. Therein, 2D geometric isomorphism was created to extend the $H_{\infty}$-norm and the small-gain theorem into the mode-frequency domain.

Consider a proper dynamics $\hat{P}$ with the 2D transfer function $P(\lambda, s)$ that is Hurwitz. The 2D- $H_{\infty}$ norm of $P$ is defined by:

$$
\|P\|_{\infty} \equiv \max _{\lambda \in \Lambda, \omega \in \Re^{+}}|P(\lambda, j \omega)|
$$

For a matrix-valued dynamics $\hat{P}$ with all entries being proper dynamics, the $2 \mathrm{D}-H_{\infty}$ norm of $P$ is defined by:

$$
\|P\|_{\infty} \equiv \max _{\lambda \in \Lambda, \omega \in \Re^{+}} \bar{\sigma}(P(\lambda, j \omega))
$$

where $\bar{\sigma}$ denotes the singular value of the underlying matrix. It can be proved that bounded $2 \mathrm{D}-H_{\infty}$ norm implies exponential decay, passivity and dissipativity for any proper dynamics.

Suppose a nominal dynamics $M$ is feedback-connected by an unstructured uncertainty $\Delta$ bounded by $2 \mathrm{D}-H_{\infty}$ norm, $\|\Delta\|_{\infty} \leq \gamma^{-1}$, as shown in Figure 1, then the closed-loop dynamics is guaranteed to be well-posed and internally stable if and only if $\|M\|_{\infty}<\gamma$. This is named 2D Small Gain Theorem.

Figure 1. For 2D Small Gain theorem.

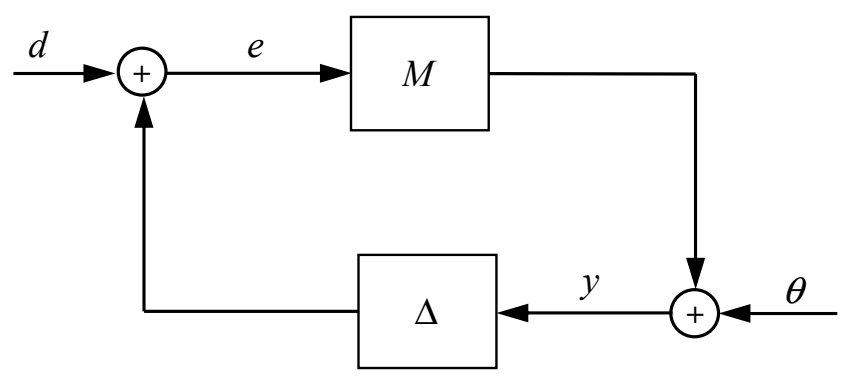

\section{Robust Performance}

Based on the 2D Small Gain theorem in Section 3, we will formulate in this section the sufficient and necessary condition of robust performance for the distributed control in mode-frequency domain.

Accommodating some extent of model uncertainties, the mode-frequency responses can be curve-fitted in the mode-Bode plot as a dense set:

$$
\left\{G_{0}\left(1+W_{1} \Delta_{1}\right):\left\|\Delta_{1}\right\|_{\infty} \leq 1\right\} .
$$

Therein $G_{0}$ stands for a nominal plant: 


$$
G_{0}(\lambda, s)=\frac{\tau_{0} s+1}{\tau_{0} s^{2}+s+\lambda}
$$

where the nominal value of heat-diffusion relaxation $\tau_{0}$ is assigned to be uniformly distributed. The 2D robustness weighting $W_{1}$ envelops all multiplicative perturbations $W_{1} \Delta_{1}$ in the mode-Bode magnitude plot. As such, the dense set in Equation (15) contains all mode-frequency responses considered in real operation.

As shown in Figure 2a, the 2D controller $K$, pre- or post-composite to the plant, together with a negative unit feedback -1 executes the feedback compensation. The controller $K$ is to guarantee specified performance for any member of the dense set in Equation (15), named Robust Performance. In $L_{2}$-gain control, the performance is specified by:

$$
\int_{0}^{T} \int_{\Omega} C(x)\left|\hat{z}_{2}(x, t)\right|^{2} d x d t<\gamma^{2} \int_{0}^{T} \int_{\Omega} C(x)\left|\hat{w}_{2}(x, t)\right|^{2} d x d t, \forall T \in[0, \infty),
$$

where the exogenous disturbances $w_{2}$ comprises the slow-time reference command and sensor noises of higher frequencies, and the tracking error $e$ has been weighted by the performance weighting $W_{2}$, i.e., $z_{2}=W_{2} e$.

Figure 2. (a) Closed-loop demanding for robust performance; (b) Robust stability of Figure 2a; (c) Fractional transformation of Figure $2 b$.

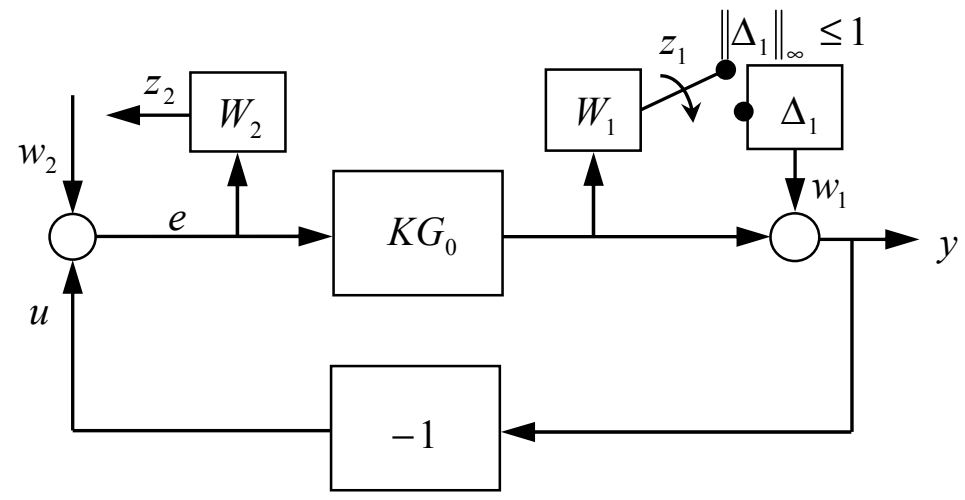

(a)

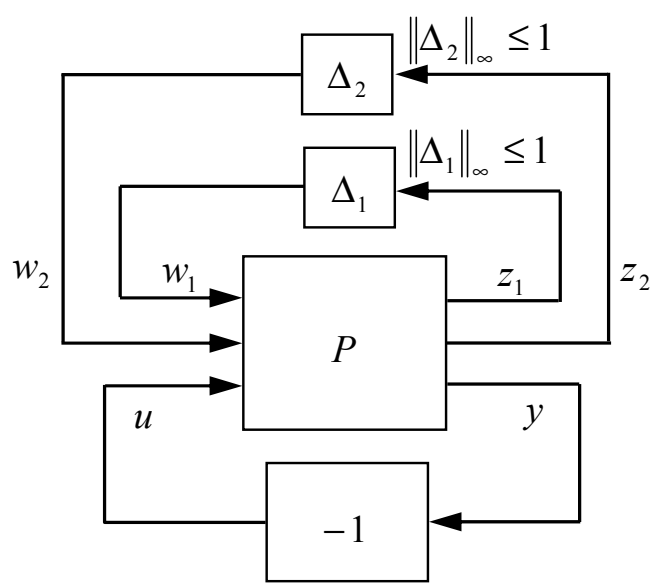

(b)

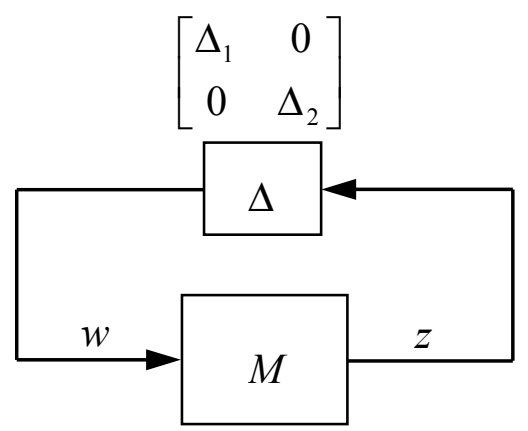

(c) 
Tracing the path of signal flow in Figure 2a, one can transform the interconnection of blocks in Figure $2 \mathrm{a}$ into the feedback-interconnection of three blocks:

$$
\text { Generalized plant } P:\left[\begin{array}{c}
z_{1} \\
z_{2} \\
y
\end{array}\right]=\left[\begin{array}{ccc}
0 & W_{1} K G_{0} & W_{1} K G_{0} \\
0 & W_{2} & W_{2} \\
1 & K G_{0} & K G_{0}
\end{array}\right]\left[\begin{array}{c}
w_{1} \\
w_{2} \\
u
\end{array}\right] \text {; }
$$

External feedback: $u=-y$;

$$
\text { Internal feedback 1: } w_{1}=\Delta_{1} z_{1},\left\|\Delta_{1}\right\|_{\infty} \leq 1 \text {. }
$$

Therein the internal disturbance $w_{1}$ and stability variable $z_{1}$ are induced from modelling uncertainty $\Delta_{1}$ . Moreover, if we replace the $L_{2}$-gain performance in Equation (17) by an internal feedback such as:

$$
\text { Internal feedback 2: } w_{2}=\Delta_{2} z_{2},\left\|\Delta_{2}\right\|_{\infty} \leq 1,
$$

then the robust performance of the original setting in Figure 2a is equivalent to the robust stability of the closed-loop system in Figure 2b. This equivalency is inferred from 2D Small Gain theorem in conjunction with the equivalence of $L_{2}$-gain to 2D- $H_{\infty}$ norm. Substituting Equation (19) into Equation (18) yields the lower fractional transformation of Figure $2 b$, as shown in Figure 2c. It becomes the feedback-interconnection of two blocks:

$$
M=\left[\begin{array}{ll}
-W_{1} T & W_{1} T \\
-W_{2} S & W_{2} S
\end{array}\right] \text { and } \Delta=\left[\begin{array}{cc}
\Delta_{1} & 0 \\
0 & \Delta_{2}
\end{array}\right] ; z=\left[\begin{array}{l}
z_{1} \\
z_{2}
\end{array}\right], w=\left[\begin{array}{l}
w_{1} \\
w_{2}
\end{array}\right],
$$

where $S$ is the sensitivity function $1 /\left(1+K G_{0}\right)$ and $T$ is its complementary function, i.e., $S+T=1$. The first requirement of the controller $K$ is to make the sensitivity function $S$ asymptotically stable.

If the $\Delta$-structured singular value $\mu_{\Delta}$ is defined by:

$$
\mu_{\Delta}(M)=\frac{1}{\min \{\bar{\sigma}(\Delta): \operatorname{det}(I-M \Delta)=0\}},
$$

then based on Nyquist criterion, the robust 2D-stability of Figure 2c is guaranteed, so is robust performance of Figure 2a, if and only if:

$$
\mu_{\Delta}(M(\lambda, j \omega)) \leq 1, \text { for all }(\lambda, \omega) \in \Lambda \times \mathfrak{R}^{+} .
$$

Substituting Equation (22) into Equation (23) yields:

$$
\mu_{\Delta}(M)=\frac{1}{\min \left\{\max \left(\left|\Delta_{1}\right|,\left|\Delta_{2}\right|\right): 1+W_{1} T \Delta_{1}-W_{2} S \Delta_{2}=0\right\}},
$$

which, after careful calculation, is explicitly shown to be:

$$
\mu_{\Delta}(M)=\left|W_{1} T\right|+\left|W_{2} S\right| .
$$

Therefore, the set of sensitivity functions with robust performance is:

$$
\left\{S:\left|W_{1}(\lambda, j \omega)(1-S(\lambda, j \omega))\right|+\left|W_{2}(\lambda, j \omega) S(\lambda, j \omega)\right|<1, \forall(\lambda, \omega) \in \Lambda \times \Re^{+}\right\} .
$$

Finally, we know that any feasible loop $S$ is a stable transfer-function and a member of Equation (27). A graphical interpretation of robust performance is shown in Figure 3, where the line segment of $S+T=1$ 
inside the diamond of $\mu_{\Delta}(M)=1$ represents the set of all feasible loops $S$. In Figure 3, we also demonstrate the conservatism of singular value $\bar{\sigma}(M)$ and the risk of spectral radius $\rho(M)$ as metrics of robust performance. In fact, $\mathrm{nD}$ state-space robust control syntheses in the control literature always takes the singular value $\bar{\sigma}(M)$ as the metric of robust performance, therefore ensuring conservatism when numerically solving distributed control.

Figure 3. Graphical interpretation of robust performance.

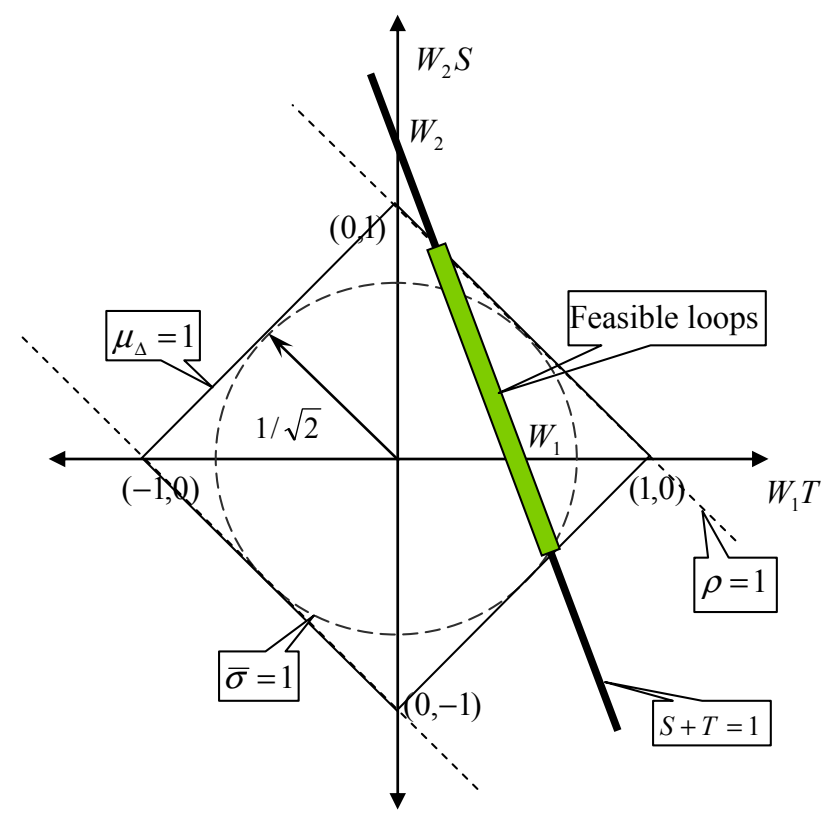

\section{2D- $\mu$ Manifolds}

The set of feasible loops $S$ 's at a fixed $(\lambda, \omega)$ :

$$
\left\{S:\left|W_{1}(1-S)\right|+\left|W_{2} S\right|<1\right\}
$$

as shown in Equation (27), is an open, convex and complex-valued set. It appears as a plate in the Cartesian plane coordinated by $\operatorname{Re}(S)$ and $\operatorname{Im}(S)$, named $\mu$-plate at $(\lambda, \omega)$. The $\mu$-plate is attributed to the following geometry:

(P1) Every $\mu$-plate is symmetric to the $\operatorname{Re}(S)$ axis.

(P2) If $\left|W_{1}\right| \geq\left|W_{2}\right|$ and both are not greater than 1, the $\mu$-plate is contained in the ellipse that has focuses $(0,0)$ and $(1,0)$, major-axis length $1 /\left|W_{2}\right|$, and minor-axis length $\sqrt{1-\left|W_{2}\right|^{2}} /\left|W_{2}\right|$.

(P3) If $\left|W_{2}\right| \geq\left|W_{1}\right|$ and both are not greater than 1, the $\mu$-plate is contained in the ellipse that has focuses $(0,0)$ and $(1,0)$, major-axis length $1 /\left|W_{1}\right|$, and minor-axis $\sqrt{1-\left|W_{1}\right|^{2}} /\left|W_{1}\right|$.

(P4) If $\left|W_{1}\right|=\left|W_{2}\right|<1$, the boundary of the $\mu$-plate is just the ellipse described in (P2) or (P3).

(P5) If $\left|W_{1}\right|=\left|W_{2}\right| \approx 1$, the $\mu$-plate degenerates into a line segment between $(0,0)$ and $(1,0)$.

(P6) If $\left|W_{1}\right| \gg\left|W_{2}\right|$, the boundary of $\mu$-plate is close to a circle of radius $1 /\left|W_{1}\right|$ centered at $(1,0)$.

(P7) If $\left|W_{2}\right| \gg\left|W_{1}\right|$, the boundary of $\left(W_{1}, W_{2}\right)$ is close to a circle of radius $1 /\left|W_{2}\right|$ centered at $(0,0)$.

(P8) For $\left|W_{1}\right|$ and $\left|W_{2}\right|$ being both greater than 1 , the $\mu$-plate degenerates into disappearance. 
The geometry of $\mu$-plates reflects some trade-off principles in feedback design as follows. Firstly, a $\mu$-plate tends to disappear as the robustness weighting $W_{1}$ and the performance weighting $W_{2}$ are simultaneously magnified. This reflects the trade-off between robust stability and nominal performance. Secondly, the uncertain perturbation $W_{1} \Delta_{1}$ arising from insufficient information of thermal inductance is distributed in high-frequency domain, so $W_{1}$ is high-passed. Thus $W_{2}$ allows only for low-pass, meaning that the closed-loop system is unable to guarantee tracking of fast-time reference temperatures to some extent. This reflects the trade-off between system maneuverability and information insufficiency. Thirdly, for large $W_{1}$ and $W_{2}$, high-gain controller can diminish the sensitivity function to achieve feasibility in Equation (27), which requires powerful heater/cooler and high-bandwidth, lownoises thermal couplers. This reflects the trade-off between hardware cost-down and robust performance.

Figure 4. (a) Exemplary robustness and performance weightings; (b) The $\mu$-manifold for Figure 4a; (c) Close view of Figure $4 b$.

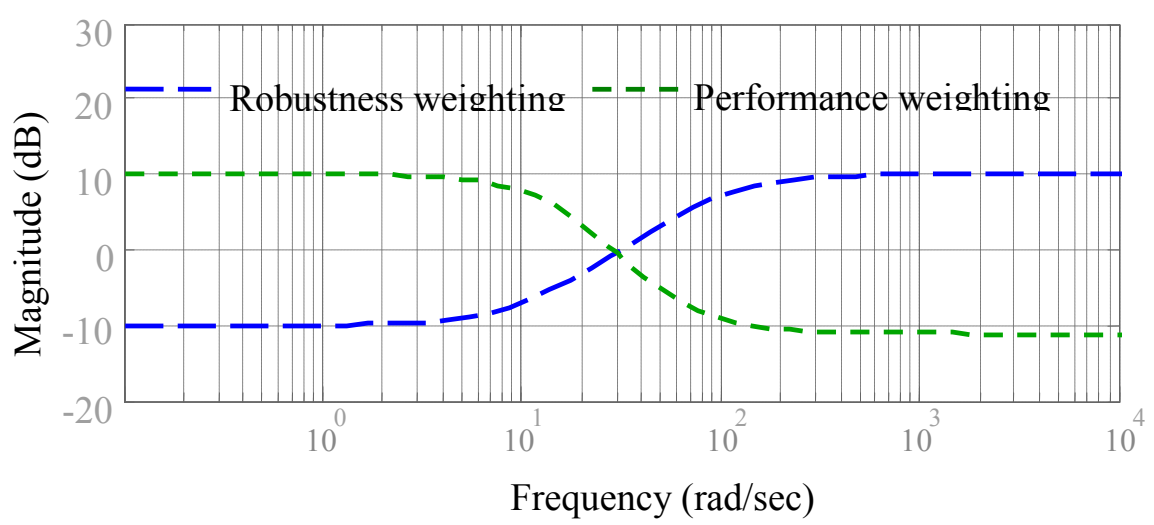

(a)

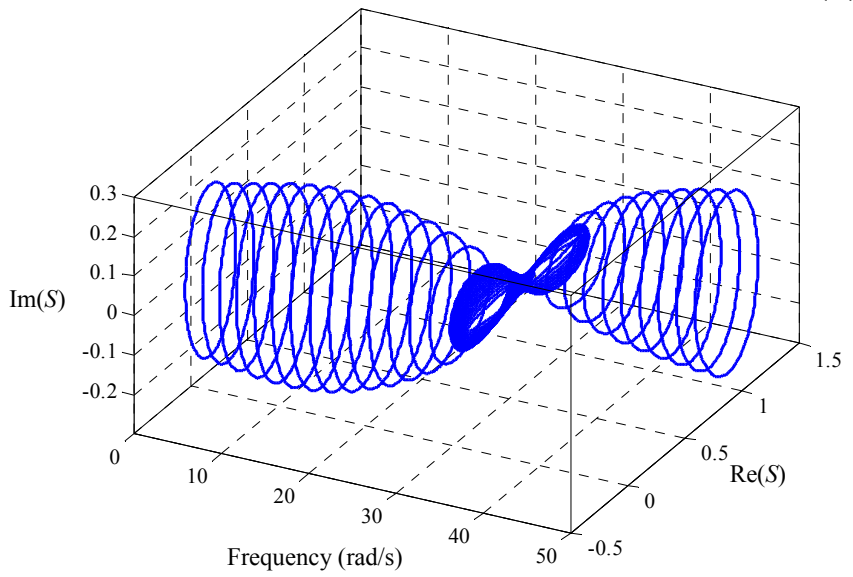

(b)

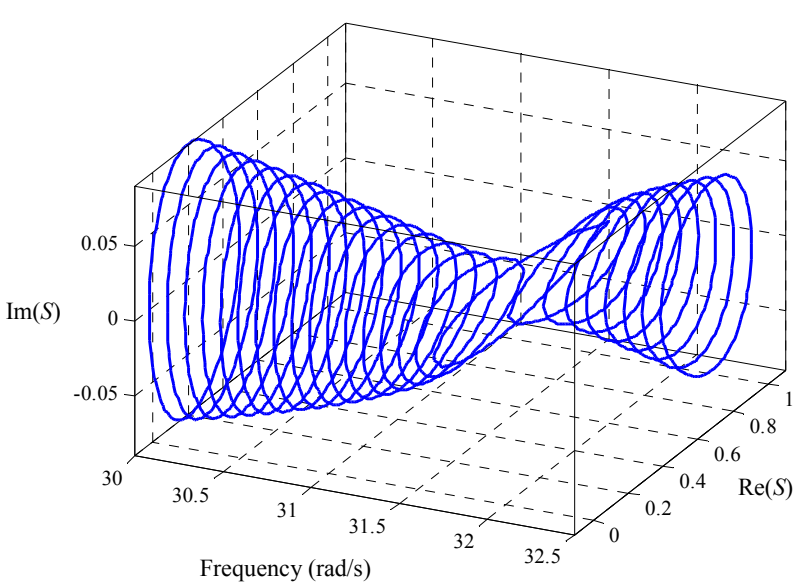

(c)

The union of all $\mu$-plates for all $\omega \in \mathfrak{R}^{+}$at fixed $\lambda$ becomes a manifold, named by $\lambda$-mode $\mu$-manifold. For a graphical manifestation of the $\mu$-plate geometry, here is plotted the $\mu$-manifold of some $\lambda$-mode for weighting functions $\left(W_{1}, W_{2}\right)$ shown in Figure $4 \mathrm{a}$. Figure $4 \mathrm{~b}$ shows the appearance of ( $\lambda$-mode) $\mu$-manifold that corresponds to the properties (P1)-(P8). For example, this $\mu$-manifold has a narrow tunnel at frequency $\omega=31.5$ at which both $W_{1}$ and $W_{2}$ have magnitudes close to one, as indicated by (P5), the close view of which is shown in Figure 4c.

The controller $K$ is proposed to take the form: 


$$
K(\lambda, s)=K_{0}(\lambda, s) \frac{\tau_{0} s^{2}+s+\lambda}{\tau_{0} s+1}
$$

where the temporal order of $K_{0}$ is larger than that of the nominator at least by one for a physically existent dynamics $K$. With the help of the $\mu$-manifold's topology, we expand 1D loopshaping technique on 2D loopshaping in mode-frequency domain to synthesize all feasible $K_{0}$ 's. After the identification of 2D performance weighting $W_{2}$ and robust weighting $W_{1}$, every feasible controller $K$ has to guarantee that every $\lambda$-mode sensitivity function map a line inside the $\lambda$-mode $\mu$-manifold for all $\lambda \in \Lambda$. In the sequel, we demonstrate the procedure of $2 \mathrm{D}-\mu$ loopshaping for non-Fourier heat conduction in longitudinal direction.

\section{2D- $\mu$ Loopshaping}

As shown in Section 2, the 2D transfer-function $G$ of non-Fourier heat conduction is:

$$
G(s, \lambda)=\frac{\tau s+1}{\tau s^{2}+s+\lambda}
$$

the typical mode-frequency response of which is shown in Figure 5 as the modal-Bode magnitude plot. Figure 5 shows that non-Fourier heat conduction in a small region behaves like a standing wave, wherein larger (smaller) modes are of higher (lower) resonant frequencies.

Figure 5. Mode-frequency response of the plant.

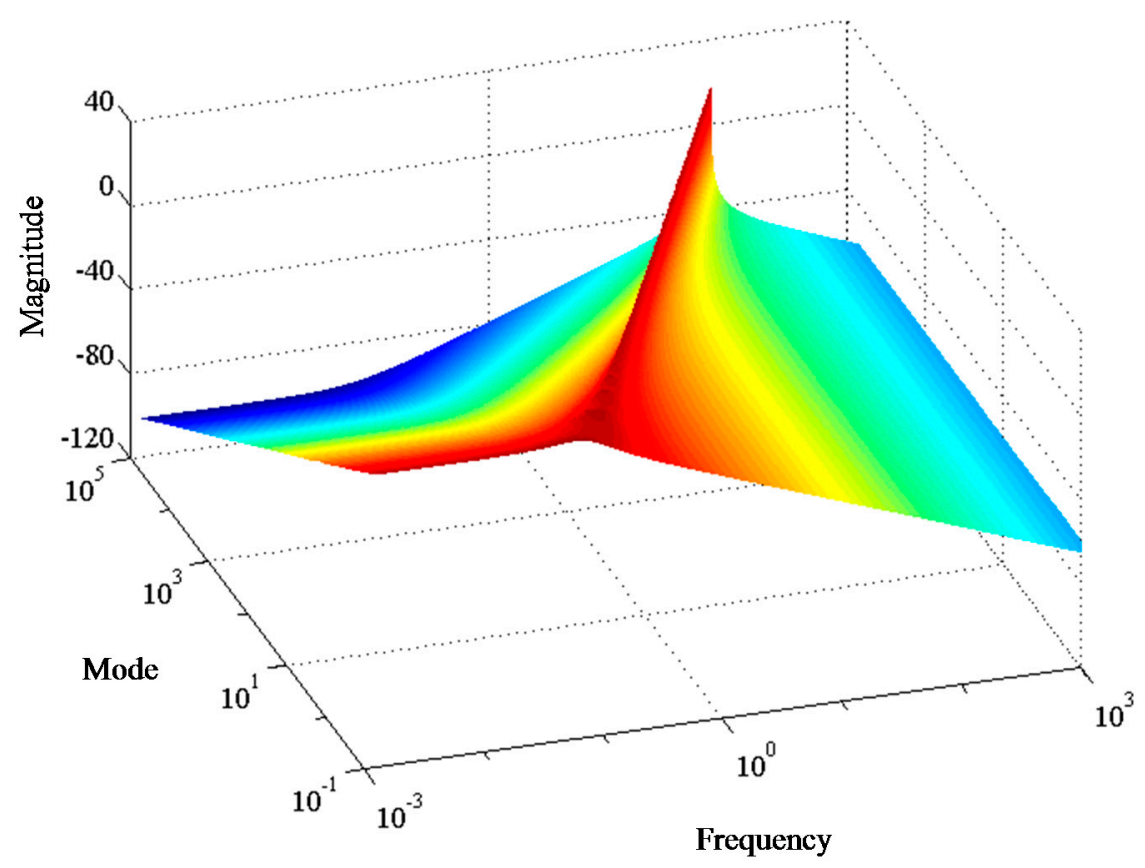

That is, with pointed control, slower heating is able to result in moderate transience, but is unable to track subtle distribution of temperature in space. On the other hand, to track a spatial distribution with larger variation, fast heating has to be adopted, but it results in severe overshoot and rising time that could burn organic materials. Figure 6 shows the mode responses at different frequencies, which provides a clearer vision of this phenomenon, wherein the mode responses at different frequencies have 
similar patterns to the frequency responses at different modes. This similarity prevents point control from reaching required temporal and spatial responses at the same time. To track precise local-temperatures and suppress the transience simultaneously, distributed control has to be developed.

Figure 6. Mode response of the plant at different frequencies.

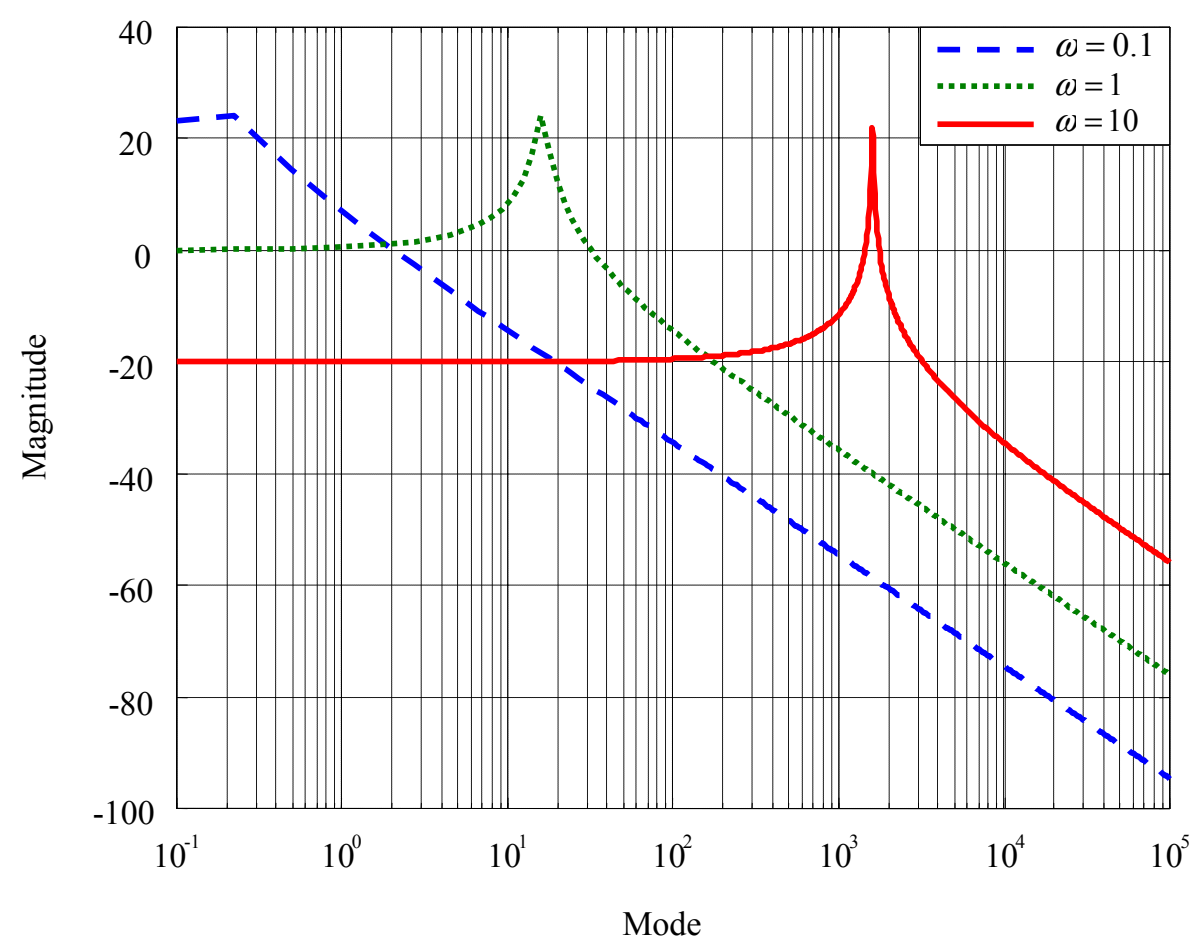

In this section, the $2 \mathrm{D}-\mu$ loopshaping in mode-frequency domain is developed to synthesize $2 \mathrm{D}$ transfer-function controllers served for distributed control. The $2 \mathrm{D}-\mu$ loopshaping consists of two main steps as follows:

Step 1. Construction of Generalized Plant

Consider that modelling uncertainties result from non-uniform distribution of the relaxation time $\tau$. Let the dense set of plants:

$$
\left\{G_{0}\left(1+W_{1} \Delta_{1}\right):\left\|\Delta_{1}\right\|_{\infty} \leq 1\right\}
$$

contain the set of mode-frequency responses accommodating empirical extent of the uncertainty, wherein $G_{0}$ stands for the nominal plant:

$$
G_{0}(\lambda, s)=\frac{\tau_{0} s+1}{\tau_{0} s^{2}+s+\lambda} .
$$

In Equation (31), $\Delta_{1}(\lambda, j \omega)$ accounts for the phase uncertainty for each mode, and the magnitude of the perturbation is specified by the $2 \mathrm{D}$ robustness weighting $W_{1}$. The perturbation satisfies:

$$
\left|\frac{G(\lambda, j \omega)}{G_{0}(\lambda, j \omega)}-1\right| \leq\left|W_{1}(\lambda, j \omega)\right|, \forall(\lambda, \omega) \in \Lambda \times \Re^{+}
$$


with a preset range of $\tau$, so the 2D robustness weighting $W_{1}$ is chosen as the envelop of all multiplicative perturbations $W_{1} \Delta_{1}$ in the modal-Bode magnitude plot. By this way, a $2 \mathrm{D}$ robustness weighting $W_{1}$ can be curve-fitted out of mode-frequency responses, as shown in Figure 7.

Figure 7. Robust and performance weightings at different modes $\left(\lambda_{n}=n^{2} / 200\right)$.

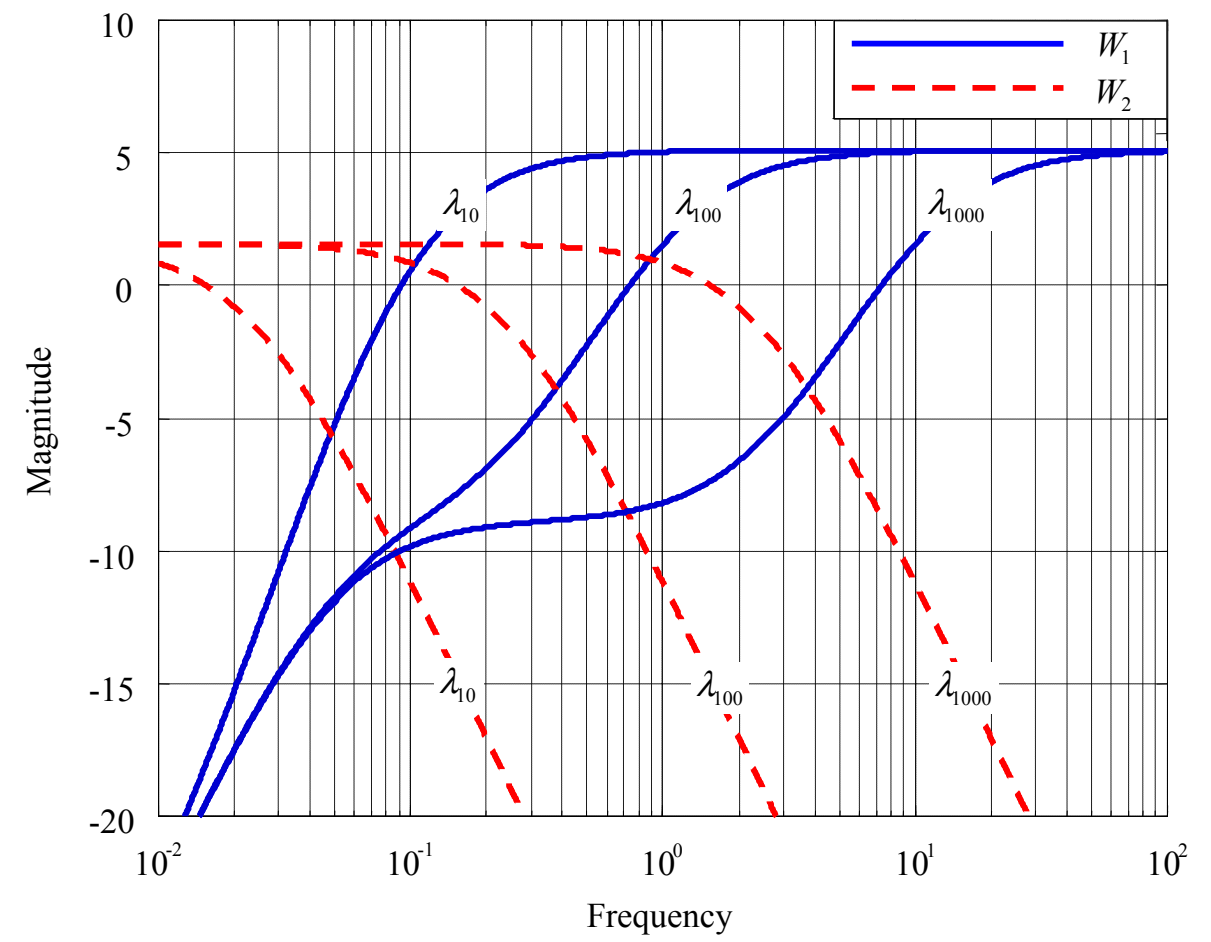

For $\tau \in[4.5,20]$, it is chosen as:

$$
W_{1}(\lambda, s)=\frac{1.8 s\left(s+0.01 \lambda^{1 / 2}\right)}{(s+0.05)\left(s+0.05 \lambda^{1 / 2}\right)}\left(\lambda \in \mathfrak{R}^{+}\right) .
$$

As for the 2D performance weighting $W_{2}$, it has to satisfy two requirements: (i) following the 2D- $\mu$ manifold's rules (P5)-(P8) in Section 5 to guarantee feasibility; and (ii) being of high-pass in space and of low-pass in time to achieve the required performance- suppress temporally abrupt transience and simultaneously track subtle temperature distribution in space. Thereby, the $2 \mathrm{D}$ performance weighting $W_{2}$ is chosen as:

$$
W_{2}(\lambda, s)=\frac{0.012 \lambda^{1 / 2}}{s+0.01 \lambda^{1 / 2}}\left(\lambda \in \Re^{+}\right) .
$$

Figure 8 shows the 2D performance weighting $W_{2}$ in the modal-Bode plot and Figure 7 shows its projection onto the mode axis, which demonstrates the requirement (ii). 
Figure 8. Modal-Bode plot of 2D performance weighting.

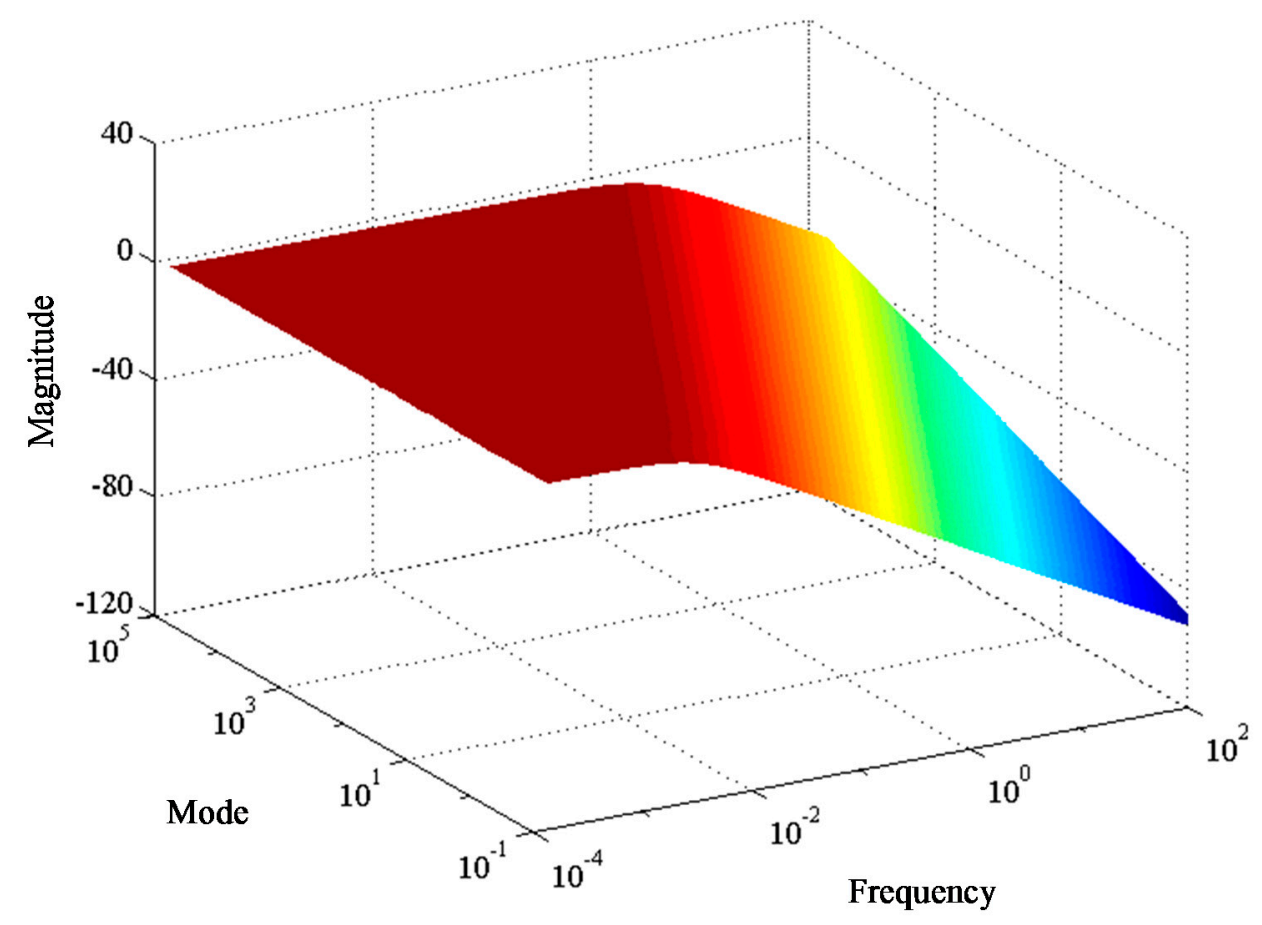

Step 2. Loopshaping Based on 2D- $\mu$ Manifold's Rules

Based on the principle of 1D- $\mu$ loopshaping in [35] and 2D- $\mu$ manifolds rules in Section 5, every loop gain $L$ of the closed-loop at each mode should satisfy:

$$
|L|>\frac{\left|W_{2}\right|}{1-\left|W_{1}\right|} \text { where }\left|W_{2}\right| \gg>1>\left|W_{1}\right| \text {, and }|L|<\frac{1-\left|W_{2}\right|}{\left|W_{1}\right|} \text { where }\left|W_{2}\right|<1<<\left|W_{1}\right| .
$$

Accordingly, we plot two curves in Bode magnitude plot: first, the graph of $\left|W_{2}\right| /\left(1-\| W_{1}||\right)$ over the low-frequency range where $\left|W_{2}\right|>1>\left|W_{1}\right|$; second, the graph of $\left(1-\left|W_{2}\right|\right) /\left|W_{1}\right|$ over the high-frequency range where $\left|W_{1}\right|>1>\left|W_{2}\right|$. Then, the graph of $|L|$ lies above the first graph at low frequency and below the second curve at high frequency. Let it roll off at least as fast as does $\left|G_{0}\right|$ at very high frequency and do a smooth transition from low to high frequency, keeping the slope as gentle as possible near crossover, the frequency where the magnitude equals 1 , to force the sensitivity function to lie inside the $\mu$-manifold.

By this way, the $2 \mathrm{D}$ loop-gain $L$ is synthesized to be:

$$
L(\lambda, s)=\frac{0.01 \lambda^{1 / 2}}{s+0.01 \lambda^{3 / 2}},
$$

which is shown in Figure 9. The 2D transfer-function controller $K$ achieving robust performance becomes:

$$
K(\lambda, s)=\frac{0.01 \lambda^{1 / 2}}{s+0.01 \lambda^{3 / 2}} \frac{\tau_{0} s^{2}+s+\lambda}{\tau_{0} s+1}
$$


which is designed to be proper not only in time but also in space on purpose of dealing with spatially discontinuous inputs.

Figure 9. Synthesis of loop gain with 2D- $\mu$ loopshaping $\left(\lambda_{n}=n^{2} / 200\right)$.

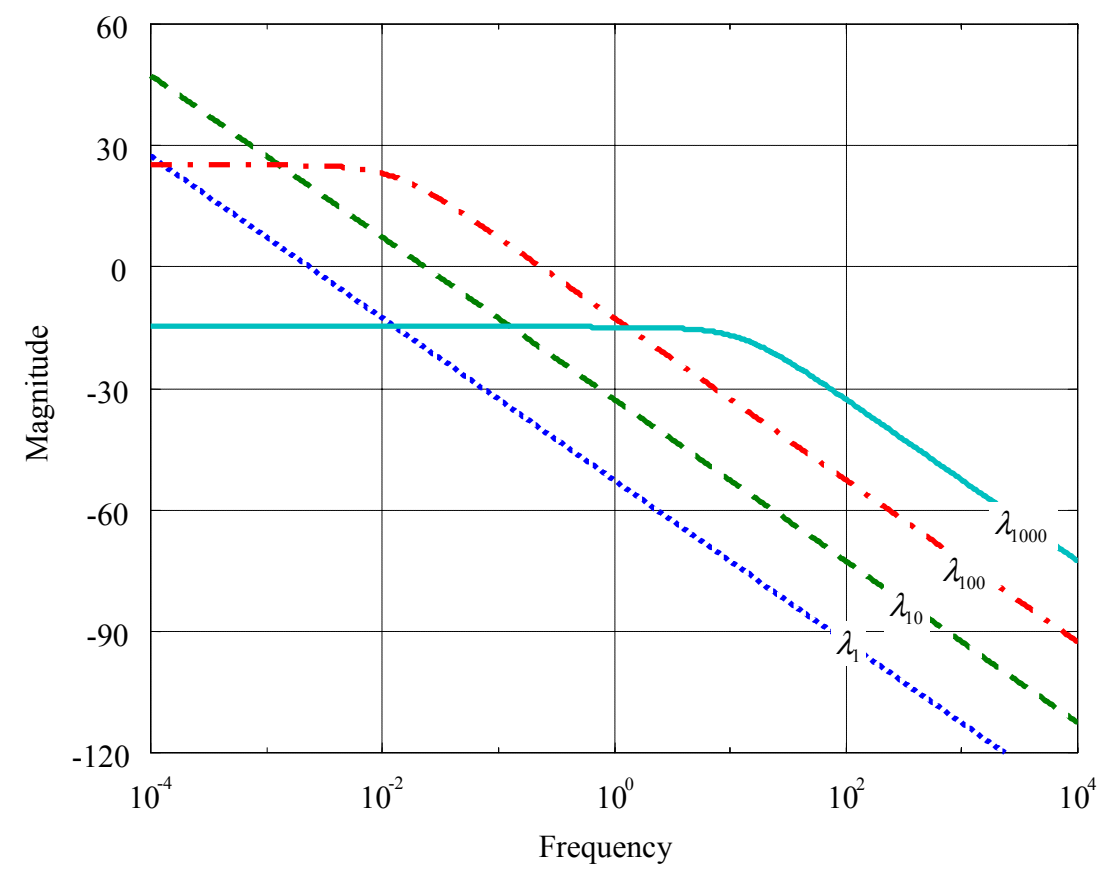

Figure 10 plots the graph of $\left|W_{1}(1-S)\right|+\left|W_{2} S\right|$, which is not larger than 1 for all modes, indicating the feasibility of the controller $K$. Finally we check the reliability of the controller $K$ in achieving robust performance by the distance of the sensitivity function to the boundary of the $\mu$-manifold. Figure 11 partly shows the reliability by plotting the sensitivity function lying inside the $\mu$-manifold at $\lambda_{100}$.

Figure 10. Feasibility of the 2D transfer function controller.

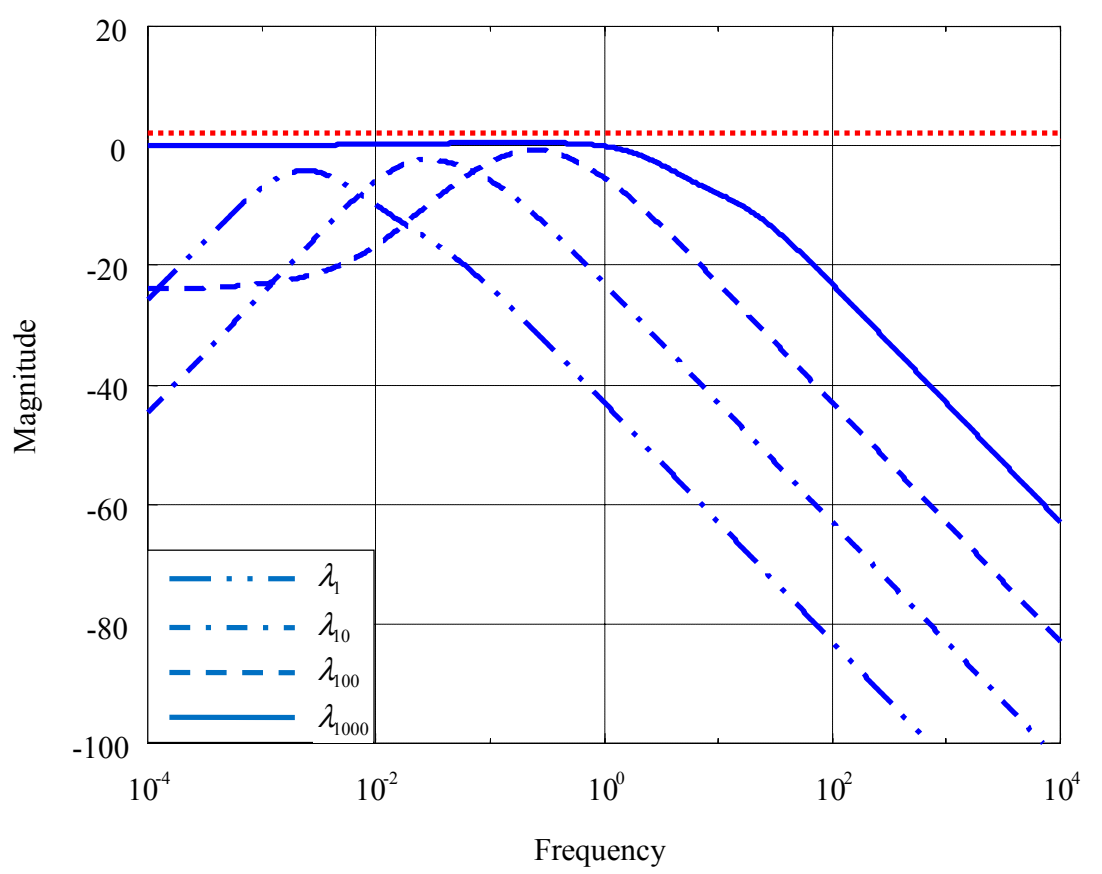


Figure 11. Sensitivity function and $\mu$-manifold at a represented mode.

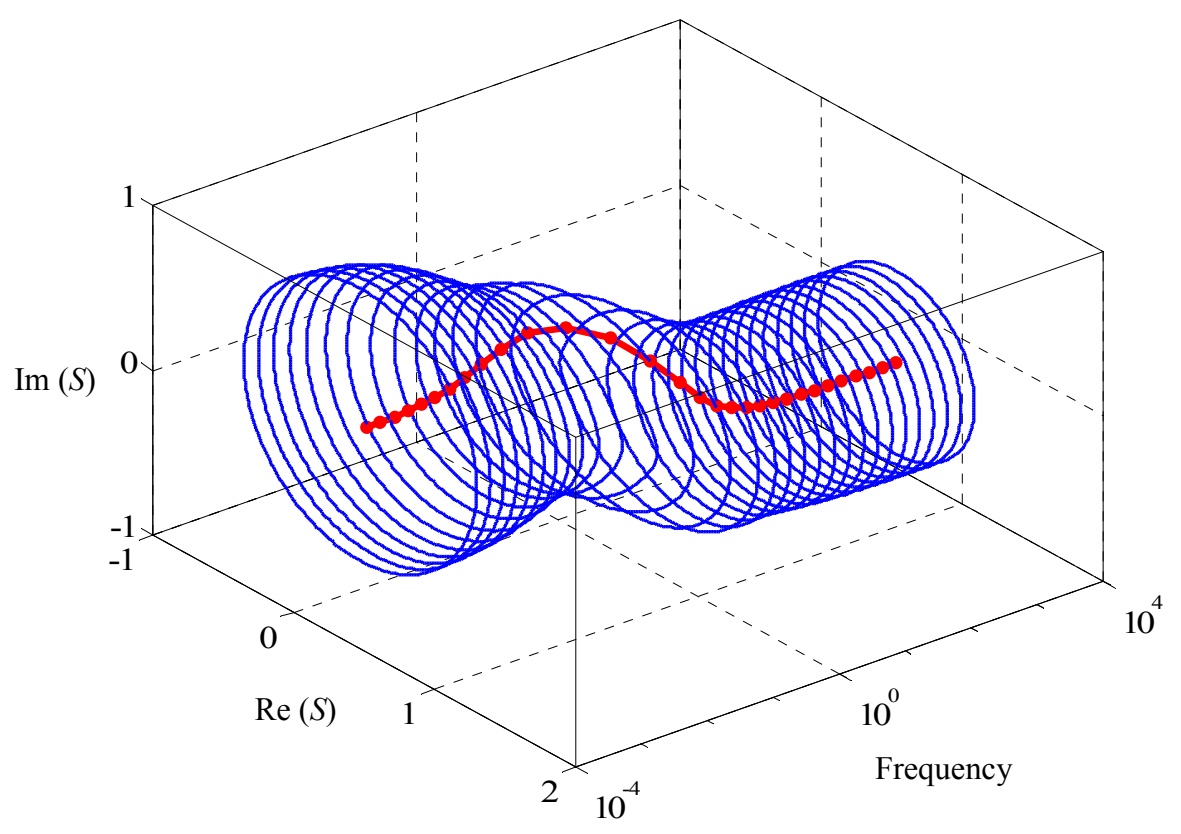

\section{Implementation of 2D Transfer-Functions Controllers}

Let a general 2D transfer-function controller $K$ be spatiotemporally discretized into some state-space realization:

$$
\begin{gathered}
v_{k+1}=\Phi v_{k}+\Gamma e_{k} \\
u_{k}=\mathcal{C} v_{k}+\mathscr{D} e_{k},
\end{gathered}
$$

for on-line signal processing in an embedded controller or for computer simulation.

Referring to Figure 12. Suppose there are $n$ state variables in Step 3, $p$ analog pins in the distributed temperature transducer and $m$ metal contacts in the distributed heater. The microcontroller array comprises $d$ dsPICs; each of them provides multi-channeled ADC converter, multi-channeled PWM generators and CAN bus protocol in peripheral, and DSP engine in CPU. With this dsPIC array, the plant output is divided into $p / d$ channels and the plant input into $\mathrm{m} / \mathrm{d}$ channels. The state $x$ is then stacked up by $n / d$ columns, $x_{i} \in \mathfrak{R}^{n / d}, i=1,2, \cdots, d$. Accordingly, the system matrices $(\Phi, \Gamma, \mathcal{C}, \mathfrak{D})$ are partitioned into $d \times d$ blocks, where:

$$
\Phi_{i j} \in \mathfrak{R}^{n / d \times n / d}, \Gamma_{i j} \in \mathfrak{R}^{n / d \times p / d}, \boldsymbol{C}_{i j} \in \mathfrak{R}^{m / d \times n / d} \text {, and } \mathfrak{D}_{i j} \in \mathfrak{R}^{m / d \times p / d} .
$$

Each $i$ th dsPIC for $i=1,2, \cdots, d$ stores the $i$ th column of present state $x_{i}$ in RAM, has the matrices $\left\{\left(\Phi_{i j}, \Gamma_{i j}, \boldsymbol{C}_{i j}, \mathscr{D}_{i j}\right): j=1,2, \cdots, d\right\}$ as parameters in ROM, and performs the computing as:

$$
u_{i}=\sum_{j=1}^{d} \boldsymbol{e}_{i j} v_{j}+\mathfrak{D}_{i j} e_{j} ; v_{i}=\sum_{j=1}^{d} \Phi_{i j} v_{j}+\Gamma_{i j} e_{j}, i=1,2, \cdots, d
$$

to update state and decide control signals, where $e$ stands for the distributed tracking error. The present states and sampled inputs distributed in the dsPIC array communicate with the built-in CAN bus peripherals. Most importantly, the Timer peripheral in every microcontroller sends interruption signals 
at a fixed time identical to the sampling time $T$ set at the stage of computer simulation to let microcontrollers' CPUs execute the DSP routines. In each Timer-interruption routine, temperatures in the distribution are sampled, the present states and measurements are communicated among microcontrollers, cloud matrix-iterated computation of Equation (41) updates the distributed states, and PWM outputs are sent to the distributed driver of the heating contacts. Such an interruption design is to guarantee that the controller under implementation is identical to that in the computer simulation. The distributed driver of the heater is an array of high-frequency switching buck choppers.

For digital implementation of 2D transfer-function controllers with the above Cloud Matrix Iteration Time-fixed DSP program (MIT) on microcontroller array, there are the following exclusive merits:

(1) We avoid a mechanical scanner for data acquisition and control output, which shortens sampling time to boost real-time fashion;

(2) Distributed computing replaces one generous-purpose microprocessor auxiliary with microcontrollers functioning DAC, PWM, and communication, therefore dramatically reducing the cost of controller manufacture without sacrificing performance; and

(3) The microcontroller array consists of the same dsPICs, which shortens the time for code development.

Figure 12. Distributed controller implementation with cloud embedded computing.

dsPIC Microcontroller array

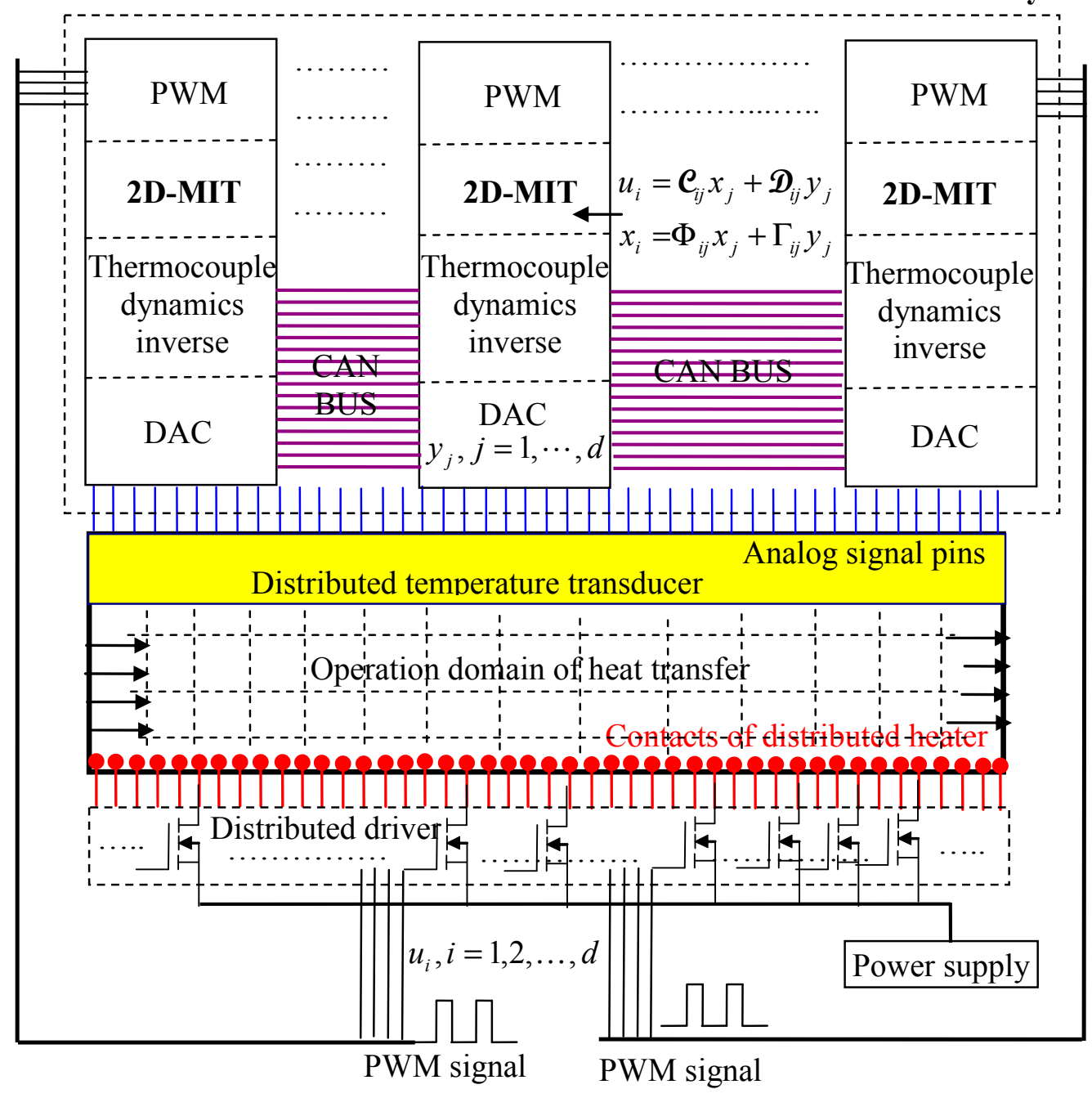




\section{Numerical and Experimental Study}

This section presents numerical and experimental investigation on the performance of the 2D transferfunction controllers that are synthesized with Section 6 and implemented by Section 7 . We will scrutinize whether the final design is capable of suppressing temporally transience and simultaneously track temperature distribution of highly spatial resolution.

Here hotdog meat is chosen as the heat-conduction material of uniform thermal parameters in the longitudinal direction. The non-Fourier heat conduction is belonging to hyperbolic dynamics:

$$
\tau \frac{\partial^{2} \psi}{\partial t^{2}}+\frac{\partial \psi}{\partial t}-\frac{k}{C} \frac{\partial^{2} \psi}{\partial x^{2}}=\tau \frac{\partial u}{\partial t}+u \quad x \in[0, \ell], \psi=0 \text { at } x=0, \ell
$$

Therein, the meat is of length $\ell=5 \mathrm{~mm}$, thermal conductivity is $k=0.8 \mathrm{~W} / \mathrm{m} \cdot \mathrm{K}$, mass density $\rho=1230 \mathrm{~kg} / \mathrm{m}^{3}$, thermal capacitance at constant volume $C_{v}=4.66 \mathrm{~kJ} / \mathrm{kg} \cdot \mathrm{K}$, and relaxing time $\tau=16 \mathrm{~s}$. Accordingly, the $2 \mathrm{D}$ transfer-function of the plant is:

$$
G(s, \lambda)=\frac{\tau s+1}{\tau s^{2}+s+\lambda}
$$

During control synthesis with $2 \mathrm{D}-\mu$ loopshaping in mode-frequency domain, we assume that modelling uncertainties arise from the parametric error of relaxing time $\tau \in[4.5,20]$, and the robust weighting is identified as above to be:

$$
W_{1}(\lambda, s)=\frac{1.8 s\left(s+0.01 \lambda^{1 / 2}\right)}{(s+0.05)\left(s+0.05 \lambda^{1 / 2}\right)} .
$$

As for the performance weighting $W_{2}$, three cases are investigated:

Case 1: $W_{2}(\lambda, s)=1.2$ that is spatiotemporally uniform;

Case 2: $W_{2}(\lambda, s)=\frac{0.012}{s+0.01}$ that is spatially uniform and temporally low-passed; and

Case 3: $W_{2}(\lambda, s)=\frac{0.012 \lambda^{1 / 2}}{s+0.01 \lambda^{1 / 2}}$ that is spatially high-passed and temporally low-passed.

Following the procedures of 2D- $\mu$ loopshaping in Section 6, three 2D transfer-function controllers for these cases are calculated for comparison of their performances.

The performance setup is to track pulse-width-modulated (PWM) temperature distribution, which is discontinuous in space. Here the temperature under tracking is uniformly distributed in $\ell / 4 \leq x \leq \ell / 2$. This kind of performance is needed for heat treatment in removing a tumor. Figure 13 shows the temperature distributions at steady state for three cases. Only Case 3 arrives at the requirement of precisely tracking the local temperatures in high resolution. Figure 14 shows the temporal responses at $x=2 \ell / 5$ of three cases, wherein only Case 3 suppresses the overshoot that otherwise could damage the meat under operation. The spatiotemporal response of Case 3 is recorded in Figure 15 that demonstrates temperature snapshots evolved into the command temperature from the initial. 
With these results, we verify the principle that distributed control of proper design is able to decouple temporal requirement from spatial requirement of the closed-loop performance. That is, temporal evolution and spatial distribution can be independently setup with distributed control technique developed in this paper.

Figure 13. Temperature distributions at steady state of three cases.

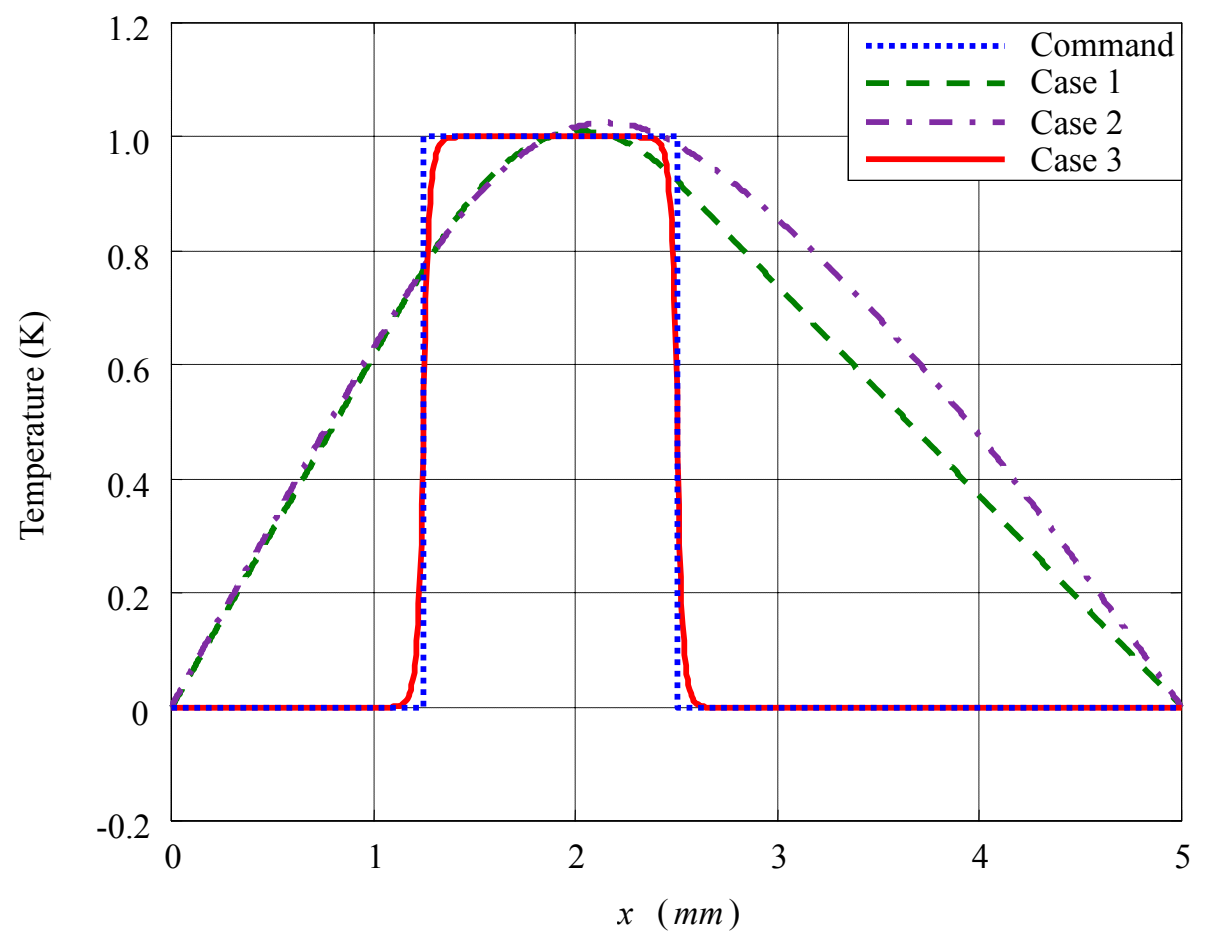

Figure 14. Temporal responses at $x=2 \ell / 5$ of three cases.

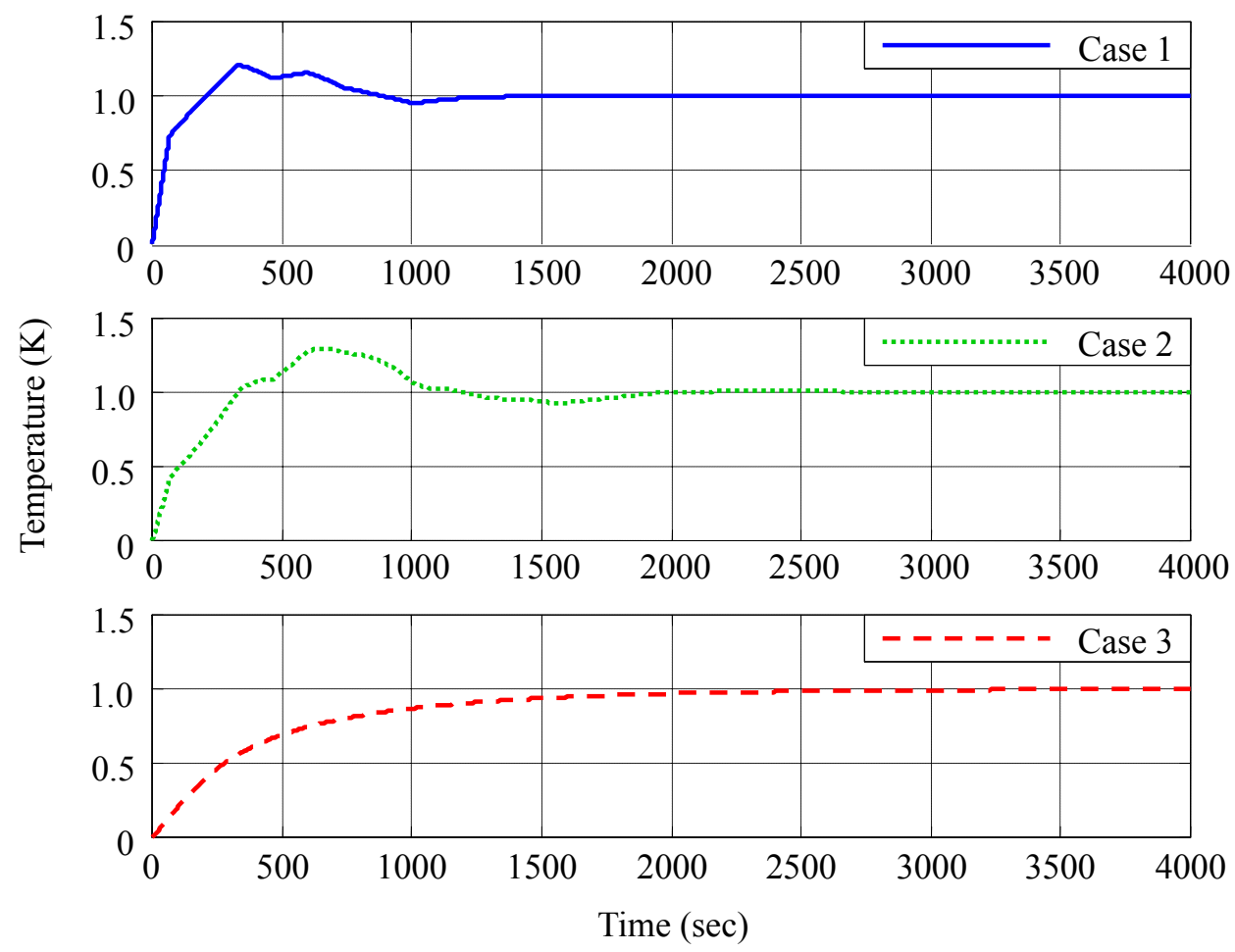


Figure 15. Temperature snapshots of Case 3.

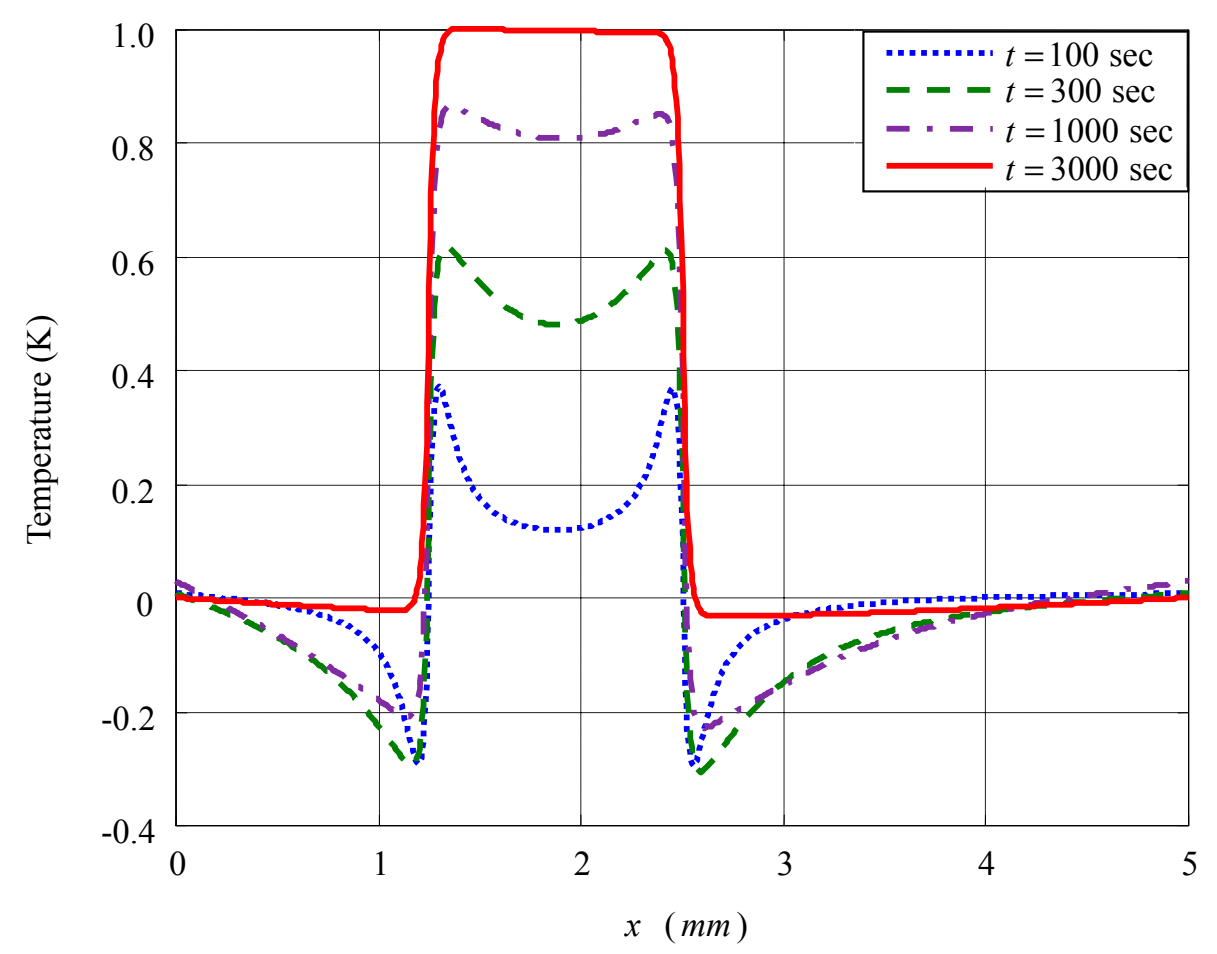

\section{Recapitulation}

Via this paper, we work on the following issues about the distributed control of heat conduction in thermally inductive materials:

(1) Verify the principle that, beyond the capability of pointed control, distributed control can simultaneously track the spatial distributions and temporal transients, which has overwhelming advantages in thermally inductive materials of modern days.

(2) Extend the classical control to $2 \mathrm{D}$ version for non-Fourier heat conduction.

(3) Implement the 2D transfer-function controller into the microcontroller array with cloud technology.

(4) Develop 2D- $\mu$ loopshaping in mode-frequency domain for the synthesis of $2 \mathrm{D}$ transfer-function controllers.

\section{Acknowledgments}

The authors give utmost thanks to National Science Council of Taiwan for financial support via the research project: NSC 102-2221-E-194-052.

\section{Author Contributions}

Boe-Shong Hong designed the research; Chia-Yu Chou and Boe-Shong Hong did the numerical experiments and all authors analyzed the data; Boe-Shong Hong wrote the paper. All authors have read and approved the final manuscript. 


\section{Conflicts of Interest}

The authors declare no conflict of interest.

\section{References}

1. Hong, B.-S.; Chou, C.-Y. Realization of thermal inertia in frequency domain. Entropy 2014, 16, 1101-1121.

2. Hong, B.-S.; Su, P.-J.; Chou, C.-Y.; Hung, C.-I. Realization of non-Fourier phenomena in heat transfer with 2D transfer function. Appl. Math. Model. 2011, 35, 4031-4043.

3. Preumont, A.; François, A.; De Man, P.; Piefort, V. Spatial filters in structural control. J. Sound Vib. 2003, 265, 61-79.

4. Preumont, A.; François, A.; De Man, P.; Loix, N.; Henrioulle, K. Distributed sensors with piezoelectric films in design of spatial filters for structural control. J. Sound Vib. 2005, 282, 701-712.

5. Brown, A.W.; Colpitts, B.G.; Brown, K. Distributed sensor based on dark-pulse Brillouin scattering. IEEE Photonics Technol. Lett. 2005, 17, 1501-1503.

6. Alahbabi, M.N.; Cho, Y.T.; Newson, T.P. 150-km-range distributed temperature sensor based on coherent detection of spontaneous Brillouin backscatter and in-line Raman amplification. J. Opt. Soc. Am. B 2005, 22, 1321-1324.

7. Fernandez, A.F.; Rodeghiero, P.; Brichard, B.; Berghmans, F.; Hartog, A.H.; Hughes, P.; Williams, K.; Leach, A.P. Radiation-tolerant Raman distributed temperature monitoring system for large nuclear infrastructures. IEEE Trans. Nucl. Sci. 2005, 52, 2689-2694.

8. Guan, Z.-G.; Chen, D.; He, S. Coherence multiplexing of distributed sensors based on pairs of fiber Bragg gratings of low reflectivity. J. Lightwave Technol. 2007, 25, 2143-2148.

9. Karnaukhova, T.V.; Pyatetskaya, E.V. Basic relations of the theory of thermoviscoelastic plates with distributed sensors. Int. Appl. Mech. 2009, 45, 660-669.

10. Karnaukhova, E.V.; Pyatetskaya, E.V. Basic equations for thermoviscoelastic plates with distributed actuators under monoharmonic loading. Int. Appl. Mech. 2009, 45, 200-214.

11. Chai, W.K.; Han, Y.; Higuchi, K.; Tzou, H.S. Micro-actuation characteristics of rocket conical shell sections. J. Sound Vib. 2006, 293, 286-298.

12. Kuang, Y.-D.; Li, G.-Q.; Chen, C.-Y. Dynamic analysis of actuator-driven circular arch of ring using impedance elements. Smart Mater. Struct. 2006, 15, 869-876.

13. Potkay, J.A.; Lambertus, G.R.; Sacks, R.D.; Wise, K.D. A low-power pressure- and temperatureprogrammable micro gas chromatography column. J. Microelectromech. Syst. 2007, 16, 1071-1079.

14. Menon, C.; Carpi, F.; Rossi, D.D. Concept design of novel bio-inspired distributed actuators for space applications. Acta Astronaut. 2009, 65, 825-833.

15. Bamieh, B.; Paganini, F.; Dahleh, M.A. Distributed control of spatially invariant systems. IEEE Trans. Autom. Control 2002, 47, 1091-1107.

16. Gorinevsky, D.M.; Stein, G. Structured uncertainty analysis of robust stability for multidimensional array systems. IEEE Trans. Autom. Control 2003, 48, 1557-1568.

17. Stewart, G.E.; Gorinevsky, D.M.; Dumont, G.A. Two-dimensional loop shaping. Automatica 2003, 39, 779-792. 
18. Stewart, G.E.; Gorinevsky, D.M.; Dumont, G.A.; Feedback controller design for a spatially distributed system: The paper machine problem. IEEE Trans. Control Syst. Technol. 2003, 11, 612628.

19. Gorinevsky, D.M.; Boyd, S.; Stein, G. Design of low-bandwidth spatially distributed feedback. IEEE Trans. Autom. Control 2008, 53, 257-272.

20. Callier, F.M.; Desoer, C.A. An algebra of transfer functions for distributed linear time-invariant systems. IEEE Trans. Circuits Syst. 1978, 25, 651-662.

21. Callier, F.M.; Winkin, J. LQ-optimal control of infinite-dimensional systems by spectral factorization. Automatica 1992, 28, 757-770.

22. Callier, F.M.; Dumortier, L.; Winkin, J. On the nonnegative self-adjoint solutions of the operator Riccati equation for infinite dimensional systems. Integr. Equ. Oper. Theory 1995, 22, 162-195.

23. Grabowski, P.; Callier, F.M. Boundary control systems in factor form: Transfer functions and inputoutput maps. Integr. Equ. Oper. Theory 2001, 41, 1-37.

24. Podlubny, I. Fractional-order systems and $P I^{\lambda} D^{\mu}$-controllers. IEEE Trans. Autom. Control 1999, 44, 208-214.

25. Valerio, D.; da Costa, J.S. Tuning of fractional controllers minimising $\mathrm{H}_{2}$ and $\mathrm{H}_{\infty}$ norms. Acta Polytech. Hung. 2006, 3, 55-70.

26. Vinagre, B.M.; Feliu, V. Optimal fractional controllers for rational order system: A special case of the Wiener-Hopf spectral factorization method. IEEE Trans. Autom. Control 2007, 52, 2385-2389.

27. Monje, C.A.; Vinagre, B.M.; Feliu, V.; Chen, Y.-Q. Tuning and auto-tuning of fractional order controllers for industry applications. Control Eng. Pract. 2008, 16, 798-812.

28. Padula, F.; Visioli, A. Tuning rules for optimal PID and fractional-order PID controllers. J. Process Control 2011, 21, 69-81.

29. Valcher, M.E. On the internal stability and asymptotic behavior of 2-D positive systems. IEEE Trans. Circuits Syst. 1997, 44, 602-613.

30. Valcher, M.E. State-space descriptions and observability properties of 2D finite-dimensional autonomous behaviors. Syst. Control Lett. 2001, 44, 91-102.

31. Fornasini, E.; Valcher, M.E. Controllability and reachability of 2-D positive system: A graph theoretic approach. IEEE Trans. Circuits Syst. 2005, 52, 576-585.

32. Lancaster, P.; Zaballa, I. Diagonalizable quadratic eigenvalue problems. Mech. Syst. Signal Process. 2009, 23, 1134-1144.

33. Carlos, J.; Anaya, Z. Diagonalization of quadratic matrix polynomials. Syst. Control Lett. 2010, 59, $105-113$.

34. Shu, Z.; Lam, J.; Gao, H.; Du, B.; Wu, L. Positive observers and dynamic output-feedback controllers for interval positive linear systems. IEEE Trans. Circuits Syst. 2008, 55, 3209-3222.

35. Doyle, J.; Francis, B.; Tannenbaum, A. Feedback Control Theory; Macmillan: New York, NY, USA, 1992.

36. Dahiya, R.S.; Vinayagamoorthy, M. Laplace transform pairs of n-dimensions and heat conduction problem. Math. Comput. Model. 1990, 13, 35-50.

37. Eltayeb, H.; Kılıçman, A. A note on solutions of wave, Laplace's and heat equations with convolution terms by using a double Laplace transform. Appl. Math. Lett. 2008, 12, 1324-1329. 
38. Rabenstein, R.; Trautmann, L. Multidimensional transfer function models. IEEE Trans. Circuits Syst. 2002, 49, 852-861.

39. Rabenstein, R.; Trautmann, L. Digital sound synthesis of string instruments with the functional transformation method. Signal Process. 2003, 83, 1673-1688.

40. Hong, B.-S.; Chou, C.-Y.; Lin, T.-Y. 2D transfer function modeling of thermoacoustic vibration engines with boundary heat-flux control. Asian J. Control 2013, 15, 1666-1676.

41. Hong, B.-S. Orthonormal decomposition of signals in eigen-decomposed linear systems. Math. Comput. Model. 2003, 37, 1297-1306.

42. Hong, B.-S. Construction of 2D isomorphism for 2D $H_{\infty}$-control of Sturm-Liouville systems. Asian J. Control 2010, 12, 187-199.

43. Young, N. An Introduction to Hilbert Space; Cambridge University Press: Cambridge, UK, 1988.

(C) 2014 by the authors; licensee MDPI, Basel, Switzerland. This article is an open access article distributed under the terms and conditions of the Creative Commons Attribution license (http://creativecommons.org/licenses/by/3.0/). 